\title{
EFTUD2 gene deficiency disrupts osteoblast maturation and inhibits chondrocyte differentiation via activation of the p53 signaling pathway
}

Jing Wu ${ }^{1}$, Yi Yang ${ }^{2}$, You He${ }^{3}$, Qiang $\mathrm{Li}^{4}, \mathrm{Xu} \mathrm{Wang}^{5}$, Chengjun Sun ${ }^{1}$, Lishun Wang ${ }^{6}, \mathrm{Yu} \mathrm{An}^{7^{*}}$ and Feihong Luo ${ }^{1^{*}}$

\begin{abstract}
Background: Mandibulofacial dysostosis with microcephaly (MFDM) is characteristic of multiple skeletal anomalies comprising craniofacial anomalies/dysplasia, microcephaly, dysplastic ears, choanal atresia, and short stature. Heterozygous loss of function variants of EFTUD2 was previously reported in MFDM; however, the mechanism underlying EFTUD2-associated skeletal dysplasia remains unclear.

Results: We identified a novel frameshift variant of EFTUD2 (c.1030_1031delTG, p.Trp344fs*2) in an MFDM Chinese patient with craniofacial dysmorphism including ear canal structures and microcephaly, mild intellectual disability, and developmental delay. We generated a zebrafish model of eftud2 deficiency, and a consistent phenotype consisting of mandibular bone dysplasia and otolith loss was observed. We also showed that EFTUD2 deficiency significantly inhibited proliferation, differentiation, and maturation in human calvarial osteoblast (HCO) and human articular chondrocyte (HC-a) cells. RNA-Seq analysis uncovered activated TP53 signaling with increased phosphorylation of the TP53 protein and upregulation of five TP53 downstream target genes (FAS, STEAP3, CASP3, $P 21$, and SESN1) both in HCO and in eftud2-/- zebrafish. Additionally, inhibition of p53 by morpholino significantly reduced the mortality of eftud2-/- larvae.

Conclusions: Our results confirm a novel de novo variant of the EFTUD2 gene and suggest that EFTUD2 may participate in the maturation and differentiation of osteoblasts and chondrocytes, possibly via activation of the TP53 signaling pathway. Thus, mutations in this gene may lead to skeletal anomalies in vertebrates.
\end{abstract}

Keywords: EFTUD2, Mandibulofacial dysostosis with microcephaly, Developmental delay, TP53 signaling pathway, Zebrafish, Osteoblast, Chondrocyte

\section{Background}

Mandibulofacial dysostosis with microcephaly (MFDM, MIM\# 610536) is a rare syndrome with a wide spectrum of congenital anomalies [1-3]. The main clinical features are characteristic facial features and associated craniofacial malformations comprising malar and mandibular hypoplasia, micrognathia, dysplastic ears, cleft palate, choanal atresia, and microcephaly, which are consequences of

\footnotetext{
* Correspondence: anyu@fudan.edu.cn; luofh@fudan.edu.cn

${ }^{7}$ Human Phenome Institute, Fudan University, 825 Zhangheng Road,

Shanghai 201203, China

1 Department of Pediatric Endocrinology and Inherited Metabolic Diseases,

Children's Hospital of Fudan University, Shanghai 201102, China

Full list of author information is available at the end of the article
}

skeletal development impairment of the head and face [1-6]. Other extracranial abnormalities, including choanaloresophageal atresia, congenital heart disease, limb defects, intellectual disability, and short stature, are frequently presented in MFDM $[1,7,8]$. As a result of skeletal dysplasia, some patients may present respiratory difficulty, hearing loss, and developmental delays $[5,6,9,10]$.

The heterozygous pathogenic variant or deletion in the elongation factor Tu GTP-binding domain-containing 2 gene (EFTUD2, MIM\# 603892) is considered the cause of MFDM [9-12]. Thus far, 95 EFTUD2 variants have been reported previously in MFDM patients, and most

(c) The Author(s). 2019 Open Access This article is distributed under the terms of the Creative Commons Attribution 4.0 International License (http://creativecommons.org/licenses/by/4.0/), which permits unrestricted use, distribution, and 
of them are de novo [11]. An animal model of eftud2 deficiency in vertebrates could mimic some clinical phenotypes of abnormal morphology, such as small head/eye and curved bodies [13]. However, the mechanism by which haploinsufficiency of EFTUD2 leads to bone deformities has yet to be explored.

In this study, we identified a novel de novo EFTUD2 null variant in a Chinese patient by whole-exome sequencing (WES) analysis. We generated a zebrafish line with eftud2 disruption to investigate the facial morphology, which presents mandibulofacial anomalies in patients, and more importantly, to determine the molecular mechanism of bone dysplasia.

\section{Materials and methods}

The details of DNA sequencing, microinjection of zebrafish embryos, TALEN efficiency testing in vitro, DNA extraction from zebrafish, real-time PCR, and Western blotting are described in the supplementary methods.

\section{Subjects and DNA extraction}

A 3-year-old boy and his family were enrolled in this study. Written informed consents were obtained from his family, and this study was approved by the ethics committee of the Children's Hospital of Fudan University in Shanghai, China. A direct physical exam, computerized tomography scan (CT), and the Wechsler Intelligence Scale for Children (WISC) were performed on the proband to verify the phenotypic characteristics of the disorder. Genomic DNA of the proband and his parents was isolated from whole blood using a Qiagen mini blood kit (Qiagen, Germany).

\section{Mutation analysis}

After whole-genome array-CGH (a-CGH) analysis and WES, variant calling was performed using NextGENe ${ }^{\circ}$ (SoftGenetics, PA, USA) with default parameters based on the FASTQ format files generated from Illumina software. Each read was aligned to the reference genome (Human 37.3, SNP135). Single nucleotide variants (SNVs) and indels were identified and saved in VCF format. These SNVs and indels were used for further interpretation analysis using Ingenuity ${ }^{\circ}$ Variant Analysis $^{\text {mim }}$ (Ingenuity Systems, CA, USA). The filter cascade was performed by common variants with MAF $>0.01$ (1000 Genomes and NHLBI GO Exome Sequencing Project), genetic analysis (dominant and recessive variants), predicted deleterious (SIFT and Polyphen2), variant annotation (OMIM, COSMIC, and TCGA), and biological context. Protein structures were modeled through the Zhang Lab online system (http://zhanglab.ccmb.med.umich.edu/).
Morpholino design and EFTUD2 gene mRNA synthesis An eftud2 morpholino (EMO) targeting a splicing site of the gene and a corresponding mismatch control morpholino (EMIS-MO) were designed (www.gene-tools.com) and synthesized: EMO 5' CAGGTTATACAATC ACCTCATCAGC 3'; EMIS-MO 5' CAGaTTATAaAATaACCTaATCAaC 3'. To identify the activation of the p53 pathway in eftud2 knockout zebrafish, a morpholino oligonucleotide corresponding to P53 (5' GCGCCA TTGCTTTGCAAGAATTG 3') was synthesized as previously described [14]. For mRNA preparation, $100 \mu \mathrm{g} / \mu \mathrm{l}$ eftud2 mRNA was obtained from the linearized PCS2+ plasmid template carrying the full-length human normal EFTUD2 cDNA, using the mMESSAGEmMACHINE Kit (Ambion, USA). Details of the injection and validation of effectiveness tested by PCR amplification using EMO1 and EMO2 primers are shown in supplementary materials.

\section{TALEN mRNA design and generation}

Three transcription activator-like effector nucleases (TALENs) were designed to disrupt the first two exons of the eftud 2 gene in zebrafish with the following sequences: eftud2-T1: 5'-TGATCTTTATGACGAGTTtg gaaattatatcggACCAGAGCTGGACTCTG-3'; eftud2-T2: 5 '-ATCGGACCAGAGCTGGActctgatgaggatgaAGAGCTAGATGCAGAGG-3'; and eftud2-T3: 5'-GGAGGTG GTTCTGCATGaggataaaaagtattatccCACTGCTGAAGA AGTGT-3'. TALEN efficiency first was tested in vitro by luciferase activity detection (seen in supplementary methods), and the final TALEN plasmids were constructed in the PCS2+ backbone. After the in vitro transcription of the TALENs using the mMESSAGE mMACHINE SP6 Transcription Kit (Ambion, USA), TALEN mRNAs were purified using phenol-chloroform methods, resuspended at a concentration of $100 \mu \mathrm{g} / \mu \mathrm{l}$ in nuclease-free $\mathrm{H}_{2} \mathrm{O}$, and stored at $-80^{\circ} \mathrm{C}$ before microinjection into zebrafish embryos ( $\mathrm{AB}$ strain).

\section{shRNA construction and viral transfection}

EFTUD2 knockdown was conducted by shRNA using the PLKO.1 purolentiviral vector. The sequences of the shRNAs targeting the EFTUD2 gene were as follows: EFTUD2-sh1 5'-GCCTCTCACAGAACCCATTAT-3'; EFTUD2-sh2 5'-CCCATTATTAAGCCAGTGAAA-3'; and EFTUD2-sh3 5'-GCTTTGCTGAAACGCCTAA TA-3'. A scrambled shRNA (shNT, 5'-GCTTTGCTGA AACGCCTAATA-3') was employed as a control. Lentiviral particles were generated using a method employed in previous studies [15]. Cells were replated in 6-well plates and transfected at $\mathrm{MOI}=3$ in the presence of $8 \mu \mathrm{g} / \mathrm{ml}$ polybrene. 


\section{Zebrafish breeding and cell cultivation}

The maintenance and microinjection of zebrafish were carried out as described in a previous study [16]. Morpholinos or TALEN mRNAs were injected into one-cell stage embryos to obtain F0 mosaics. After further outcrossing of F0 mosaics with wild type (WT) to generate heterozygous eftud2 mutant offspring (F1), we obtained the F2 generation from F1 interbreeding. This study was approved by the Ethics Committee of Children's Hospital of Fudan University ([2014] No. 115).

The human calvarial osteoblast (HCO) and human articular chondrocyte ( $\mathrm{HC}-\mathrm{a})$ primary cell lines were purchased from ScienCell Research Laboratories (ScienCell, USA). HCO cells were cultured in specific medium (ScienCell, USA) containing 5\% FBS (0025, ScienCell, USA), $1 \%$ osteoblast growth supplement (4652, ScienCell, USA), and 1\% penicillin/streptomycin (0503, ScienCell, USA), whereas $\mathrm{HC}$-a cells were cultured in medium containing 5\% FBS, 1\% chondrocyte growth supplement (4682, ScienCell, USA), and 1\% penicillin/ streptomycin, at $37^{\circ} \mathrm{C}$ in a humidified $5 \% \quad \mathrm{CO}_{2}$ atmosphere.

\section{Zebrafish and cellular alcian blue and alizarin red staining Zebrafish staining}

The cartilage and bone of zebrafish were double-stained with an acid-free solution containing alcian blue 8GX and alizarin red $\mathrm{S}$ at the same time according to a previously reported method [17] and imaged under a dissecting microscope (Zeiss, Germany).

\section{Cell staining}

$\mathrm{HCO}$ and $\mathrm{HC}-\mathrm{a}$ cells were stained with alizarin red $\mathrm{S}$ and alcian blue $8 \mathrm{GX}$ solution separately at 3 days after cell confluence. Briefly, HCO cells plated in 6-well plates were washed in PBS (phosphate buffer saline) and fixed in $4 \%$ paraformaldehyde (PFA) for $15 \mathrm{~min}$, washed again in PBS 3 times, stained with $0.5 \%$ alizarin red $\mathrm{S}$ for $1 \mathrm{~h}$, and then rinsed with $\mathrm{PBS}$. The procedure for $\mathrm{HC}-\mathrm{a}$ staining was similar to that for HCO staining, except that staining with the alcian blue $8 \mathrm{GX}$ solution was conducted for $30 \mathrm{~min}$, after which the samples were washed 3 times with 3\% glacial acetic acid for 3 min each before being washed in PBS and photographed with an inverted microscope (Leica, Germany).

\section{Proliferation assay using Cell Titer-Glo}

For this assay, $2 \times 10^{4}$ cells per well were plated in a 96well plate and transfected with lentiviruses for $5 \mathrm{~h}$ after cell adherence. The proliferation of $\mathrm{HCO}$ and $\mathrm{HC}$-a cells was assessed indirectly at 3 days before cell confluence by detecting the amount of adenosine triphosphate (ATP) in viable cells according to the manufacturer's instructions (Promega, USA). Then, $100 \mu \mathrm{l}$ of the Cell
Titer-Glo reagent was added to $100 \mu$ l of culture medium, and fluorescence was measured with a luminescence detection system (BioTek, USA).

\section{ALP activity assay}

ALP enzyme activity was determined using the Alkaline Phosphatase Activity Fluorometric Assay Kit (BioVision, USA). Briefly, cells were plated in a 6-well plate at a density of $2 \times 10^{5}$ cells per well. Cell lysates were subsequently prepared in $400 \mu \mathrm{l}$ of assay buffer at 3 days before or 2 weeks after cell confluence. After cell lysates were centrifuged at $13,000 \mathrm{~g}$ for $3 \mathrm{~min}, 110 \mu \mathrm{l}$ of sample lysates was mixed with $20 \mu \mathrm{l}$ of $0.5 \mathrm{mM} 4$ methylumbelliferyl phosphate disodium salt (MUP). A standard curve was produced based on a concentration series of $0,0.1,0.2,0.3,0.4$, and $0.5 \mathrm{nM}$ MUP. All reactions were incubated in the dark at room temperature and stopped with $20 \mu \mathrm{l}$ of stop solution. The results were read at 360-nm excitation/460-nm emission (BioTek, USA).

\section{RNA sequencing analysis}

Total RNA was isolated and purified from $\mathrm{HCO}$ cells transfected with sh2 and shNT 3 days before cell confluence was observed using the Qiagen RNeasy Mini Kit (QIAGEN, Germany), after which $7 \mu \mathrm{g}$ of total RNA was used for library preparation. The total RNA was further purified with the RNeasy Micro Kit (QIAGEN, Germany) and RNase-Free DNase Set (QIAGEN, Germany) and sequenced on the Illumina HiSeq2500 platform. Hierarchical clustering analysis of DEGs (differentially expressed genes) was performed to explore gene expression patterns between the two groups. Differentially expressed genes were determined based on the following criteria: FDR $\leq 0.05$, Fold-change $\geq 2$, with the FPKM value of each gene calculated by Cufflinks (version: 2.1.1). To analyze and acquire functional information for DEGs, gene ontology (GO) and Kyoto Encyclopedia of Genes and Genomes pathway (KEGG) enrichment analyses were performed using $R$, based on the hypergeometric distribution. Additionally, information on protein-protein interactions was evaluated with the Search Tool for the Retrieval of Interacting Genes (STRING: http://string-db.org/).

\section{Synchrotron X-ray microtomography analysis}

Synchrotron radiation X-ray microtomography (SR$\mu \mathrm{CT}$ ) was performed with the BL13W1 beam line in the Shanghai Synchrotron Radiation Facility (SSRF). Adult zebrafish were anaesthetized and fixed in 4\% PFA for a week, followed by dehydration in a series of ethanol dilutions ranging from 75 to $100 \%$ ethanol. The specimens were stored vertically in plastic tubes, which were mounted on a sample stage and imaged with an 18.0 
$\mathrm{keV}$ monochromatic X-ray. A set of radiographs $(720$ projections over $180^{\circ}$ ) was recorded using an X-ray detector system with a $3.25-\mu \mathrm{m}$ pixel size (ORCA-Flash4.0, Hamamatsu Photonics KK, and Japan). The slices were reconstructed using a filtered back-projection (FBP) algorithm, and 3D renderings were created and manipulated in VG Studio Max 2.1 software.

\section{Statistical analysis}

Quantitative data are presented as the mean \pm standard deviation and were analyzed using one-way ANOVA, followed by Tukey's multiple-range test. $P<0.05$ was considered statistically significant.

\section{Results}

\section{Clinical and genetic identification of the patient}

The proband was a G1P1 full-term boy with a birth weight of $2600 \mathrm{~g}$, although the length was unavailable, who failed to pass the initial hearing test at 3 days and the second test at 42 days old in the left ear. The boy had a hoarse cry and poor sucking reflex after birth. He began to teeth at the age of 4 months, crawl at 7 months, pull self to a standing position at 9 months, and walked alone at 1 year of age, similar to other children of the same age. However, he walked unsteadily until approximately 25 months old and ran with poor coordination at 37 months. The child presented with microcephaly (head circumference of $45 \mathrm{~cm},<\mathrm{P} 3$ ), severe micrognathia, arched eyebrows, everted lips, broad nasal bridge, abnormal ear structures with hearing loss, adenoid hypertrophy, and physical growth delay (weight of $8.6 \mathrm{~kg},<\mathrm{P} 3$; length of $92.4 \mathrm{~cm}, \mathrm{P} 10-25)$ at 37 months of age (Fig. 1a) when he first visited our clinic. Adenotonsillectomy was performed, and he also received surgical operation due to poorly formed structures of the external and middle ear, with external auditory canal stenosis on the left (Fig. 1b); by the age of 57 months, boundary developmental delay was confirmed with an intelligence quotient of 61 (verbal IQ 60, performance IQ 69) via the WISC test. Preauricular tags, cleft palate, esophageal atresia, seizures, limb symptoms, and congenital heart defects were not observed. A mental development abnormality related to a $214.11-\mathrm{kb}$ duplication in the chromosomal region Xq28 (151,557,556-151,771,66 5(hg19)) involving the GABRA3, MIR105-1, MIR767, and MIR105-2 genes was found both in the proband and his normal mother using Array-CGH, while his father did not carry this variant. There was a case (Patient 250326) reported in DECEPHER with a 371-kb duplication within the region $(151,532,990-151,904,036)$ who had conditions of delayed speech and language development and intellectual disability, and there were three reports derived from DGV by SNP array of cases carrying duplications of $197 \mathrm{~kb}$ (nssv655052), $222 \mathrm{~kb}$ (nssv657732), and $211 \mathrm{~kb}$ (nssv683872) [18]. A further WES test detected only one novel heterozygous out-of-

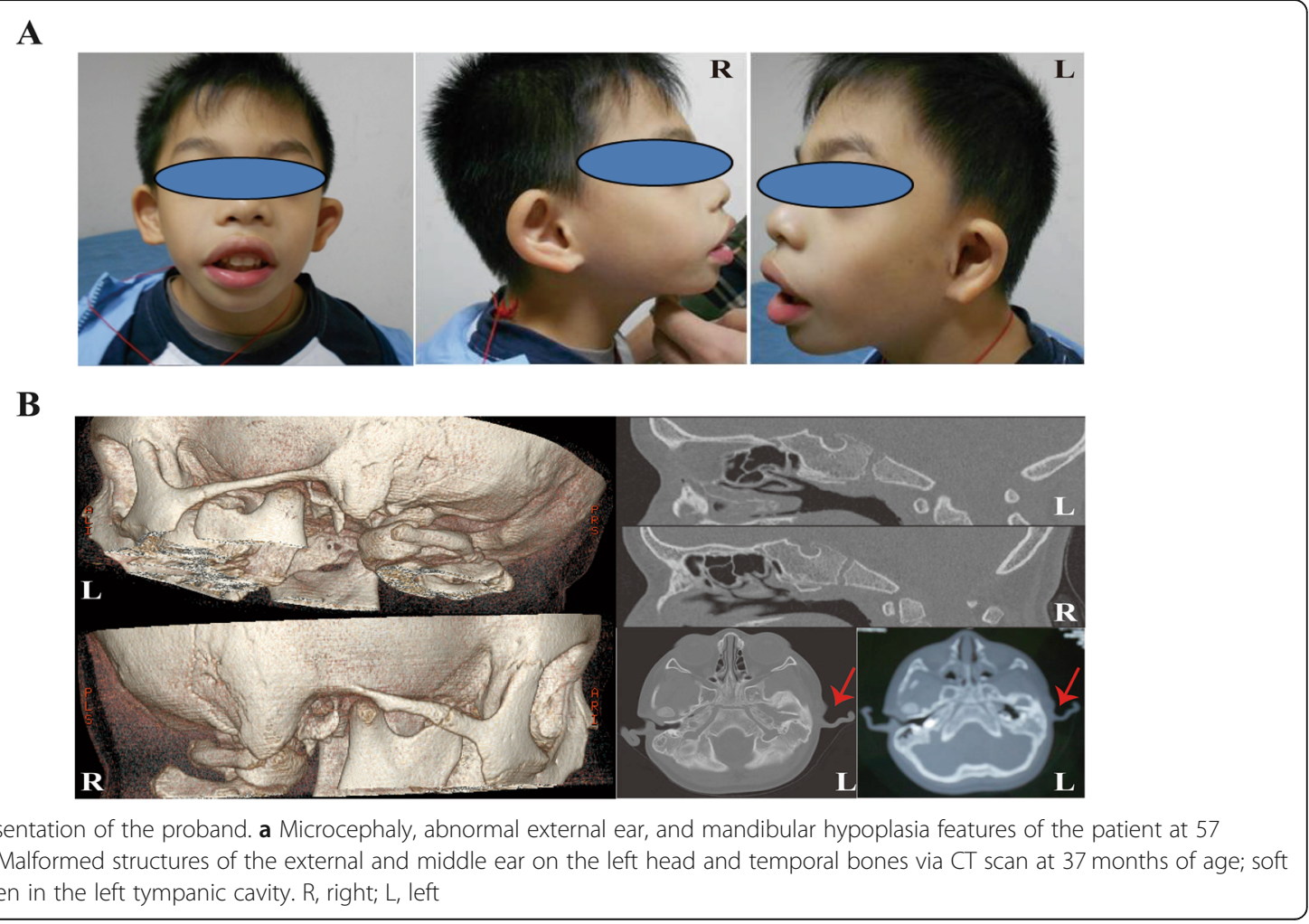

Fig. 1 Clinical presentation of the proband. a Microcephaly, abnormal external ear, and mandibular hypoplasia features of the patient at 57 months of age. $\mathbf{b}$ Malformed structures of the external and middle ear on the left head and temporal bones via CT scan at 37 months of age; soft tissue could be seen in the left tympanic cavity. R, right; L, left 
frame deletion (c.1030_1031delTG, p.Trp344fs*2) in the EFTUD2 gene (OMIM 610536), which was associated with developmental delay and craniofacial dysostosis in a biological context among 23 candidate genes by cascading filters through the Ingenuity tool, such as allele frequency below 0.01 from the available databases of 1000 Genomes, the NHLBI GO Exome Sequencing Project, and dbSNP (Fig. 2a, b and Additional file 1: Table S1). The variant truncated the wild-type $116 \mathrm{kDa}$ EFTUD2 protein into a protein of approximately $50 \mathrm{kDa}$, which is predicted to have a completely different protein structure than that of the wild type (Fig. 2c).

\section{Spatial and temporal expression of the eftud 2 gene during zebrafish development}

Wild-type zebrafish larvae were sacrificed at $24 \mathrm{hpf}, 48$ hpf, $3 \mathrm{dpf}$, and $5 \mathrm{dpf}$, and eftud 2 gene expression was analyzed by whole mount in situ hybridization (WISH) and real-time PCR. eftud2 was widely expressed throughout the embryo during early developmental stages

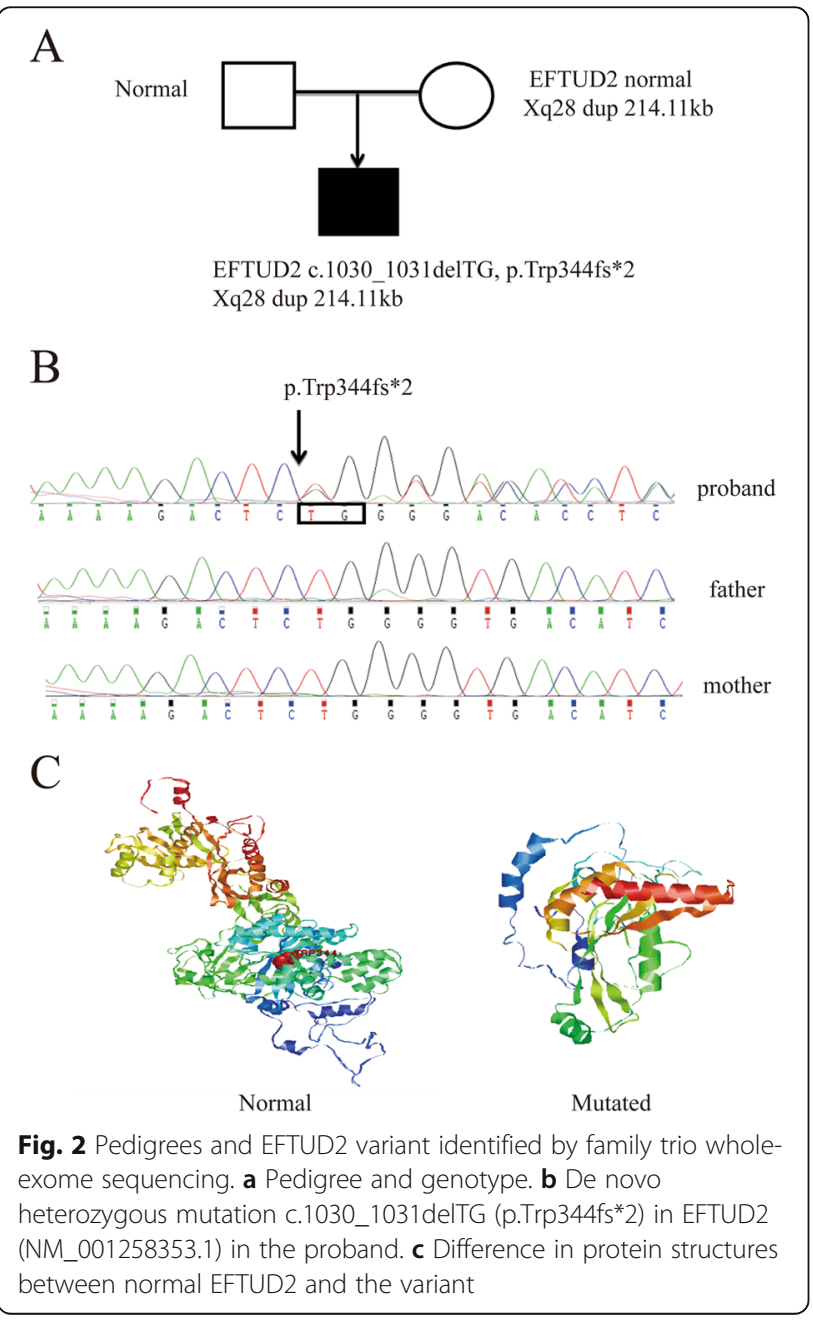

(Additional file 2: Figure S1A). eftud2 mRNA expression continued to increase until $48 \mathrm{hpf}$ and decreased thereafter (Additional file 2: Figure S1B). Among the different tissues of adult zebrafish, the eftud2 gene was expressed at high levels in the spleen, muscle, and brain, whereas it was expressed at a low level in ocular tissue (Additional file 2: Figure S1C).

\section{The efficacy of eftud2 knockdown and knockout in zebrafish}

PCR results showed that EMO could affect the normal splicing of eftud2, thus leading to its abnormal expression. As EMO is designed to target the splicing site, DNA products amplified with EMO1 from EMOinjected zebrafish larvae at 48 hpf yielded nonspecific bands, except at $250 \mathrm{bp}$, and a large fragment (1 kbp) when amplified with EMO2 (Fig. 3a). Additionally, zebrafish with a disrupted eftud2 gene exhibited curved bodies much more frequently than those injected with EMIS-MO or rescued with normal human EFTUD2 mRNA (Fig. 3b, c).

To test the effect of long-term knockout of the eftud 2 gene, targeted knockout TALENs (Additional file 3: Figure S2A, B) were established and selected through in vitro luciferase activity (Additional file 3: Figure S2C), T7E1 enzyme digestion (Additional file 3: Figure S2D), DNA sequencing (Additional file 3: Figure S2E), and Western blot assays (Fig. 3c). The successful eftud2 knockout model was confirmed via Sanger sequencing of zebrafish larvae at $3 \mathrm{dpf}$, which showed a deletion of 5 bp (c.59-63 ATGAG), and Western blot analysis showed sharply decreased protein expression (Fig. 3d, e). The deletion of $5 \mathrm{bp}(\Delta 5)$ resulted in a frameshift mutation that translated to a shortened EFTUD2 protein containing only the first 19 amino acids when compared with the reference amino acids (XP_017209079.1).

\section{eftud2 knockdown and knockout disrupted bone and cartilage development in zebrafish}

Short-term knockdown of the eftud2 gene with a specific morpholino (EMO) resulted in a significantly higher rate of curved bodies $(72 \pm 5.14 \%)$ compared with that of the EMIS-MO control group at $3 \mathrm{dpf}(10.3 \pm 4.6 \%, P<0.00$, Fig. 3b, c). The coinjection of EMO with normal human EFTUD2 mRNA, which shares $91 \%$ nucleotide homology with that of zebrafish, was employed as a rescue intervention. Rescue in EMO-treated zebrafish significantly decreased the incidence of curved larvae to $44.9 \pm 8.8 \%$ $(P<0.01$, Fig. 3c). Dysplasia of Meckel's cartilage and ceratohyals was also observed in the larvae that received an injection of EMO at $3 \mathrm{dpf}$ and $5 \mathrm{dpf}$, based on alcian blue or alizarin red staining, and the coinjection of EMO and normal human EFTUD2 mRNA could also rescue 


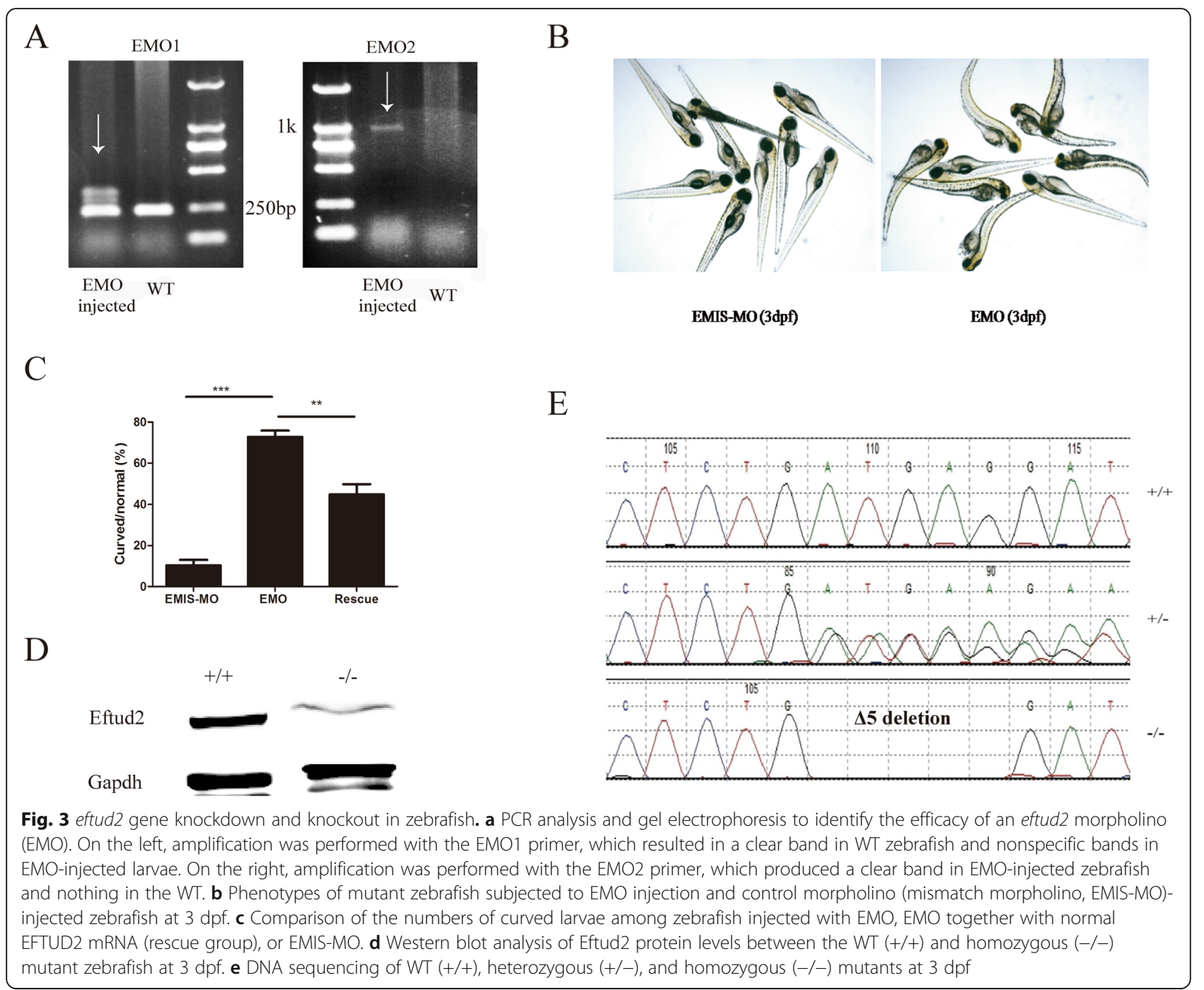

the dysgenesis in mandibular bone (Additional file 4: Figure S3A-D).

In addition to small heads and large yolks, the stable eftud2 TALEN knockout zebrafish showed obviously significant dysplasia formation in their Meckel's cartilage, ceratohyals, and ethmoid bones compared with the larvae injected with EMO at $3 \mathrm{dpf}$ and $5 \mathrm{dpf}$ (Figs. 4 and 5). Malformations in the sphenoidal sinus and notochord and even otolith loss were also recorded in the knockout larvae (Fig. $4 \mathrm{a}-\mathrm{d}$ ). Interestingly, all of the larvae with homozygous eftud2 mutation died within 5 days; therefore, only the adult zebrafish with heterozygous eftud2 mutation were available for phenotypic and genotypic analyses. The heterozygous F2-generation knockout adults exhibited a phenotype much more similar to that of human MFDM, presenting a shortened jawbone and deformity of Meckel's cartilage by synchrotron radiation $\mathrm{X}$-ray microtomography (Fig. 5a, b).
EFTUD2 gene knockdown suppressed HCO and HC-a cell growth in vitro

To determine whether the EFTUD2 gene affects osteoblast proliferation, $\mathrm{HCO}$ cells were transfected with targeting shRNAs (sh2 and sh3, Fig. $6 \mathrm{a}-\mathrm{c}$ ). HCO cell proliferation decreased by $65 \%$ after lentivirus transfection $(P<0.0001)$ compared with that after shNT treatment at 3 days before cell confluence (Fig. $6 \mathrm{c}$ and $\mathrm{k}$ ). Furthermore, the expression of relevant genes, including the alkaline phosphatase $(A L P)$, collagen type 1 alpha 1 (COL1A1), and osteopontin (OPN) genes (Fig. 5d, e, g, j), along with ALP activity (Fig. 6f), was significantly decreased throughout the proliferation and differentiation period $(P<0.01)$. COL1A1, which is a marker of the early differentiation and maturation of the extracellular matrix, showed no change before cell confluence and was decreased by $90 \%$ when entering the differentiation phase in the sh2 and sh3 groups compared with that in 

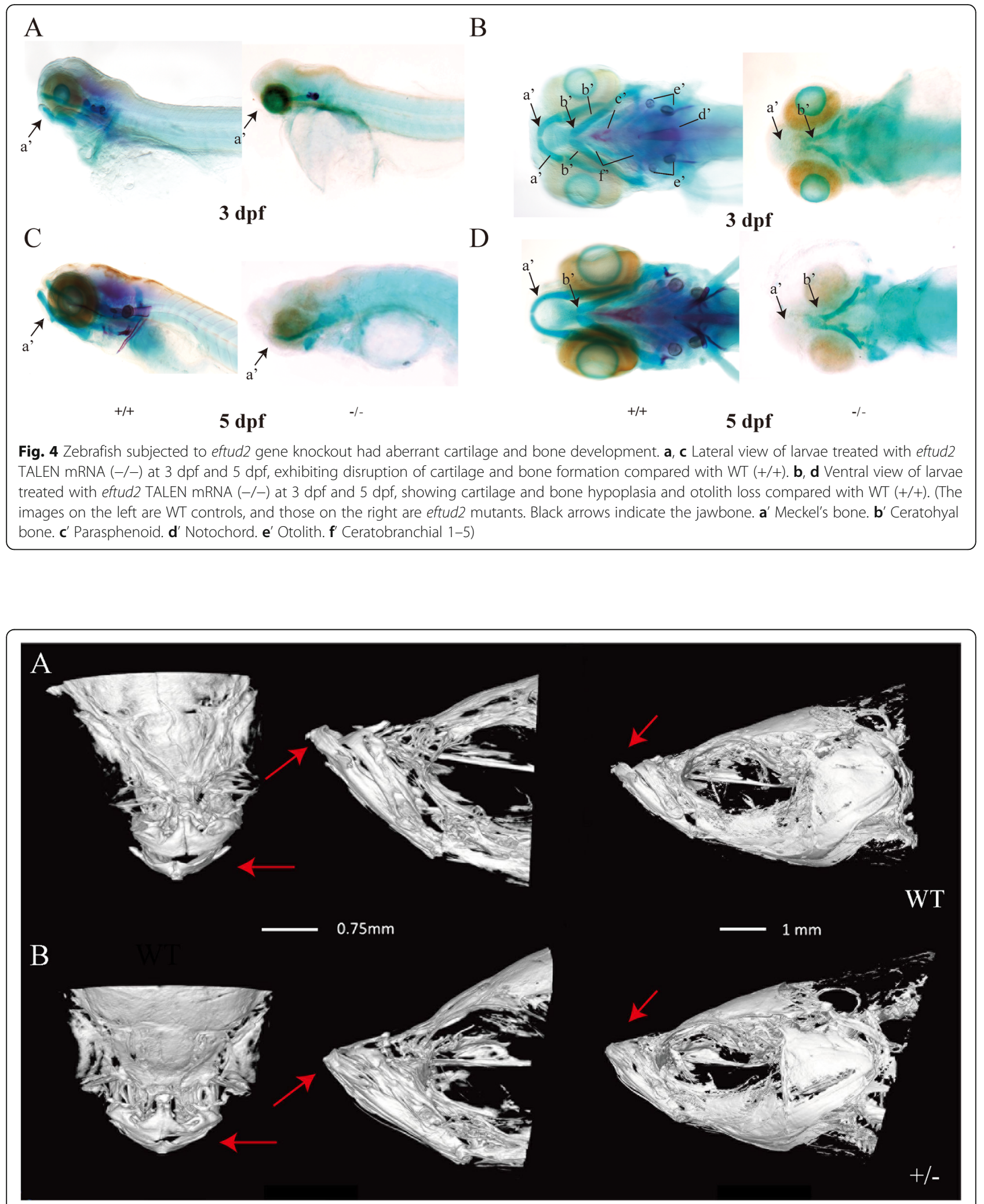

Fig. 5 Adult zebrafish with heterozygous eftud2 mutation showed abnormal mandibular bone. a Mandible bone of wild-type adults (WT) scanned by synchrotron radiation X-ray microtomography (arrows). Scale bar, $0.75 \mathrm{~mm}$. b The heterozygous F2 generation (+/-) exhibited a shortened mandibular bone (arrows). Images on the left are viewed from the dorsal side, while the images in the middle and on the right show lateral views. Scale bar, $1 \mathrm{~mm}$ 


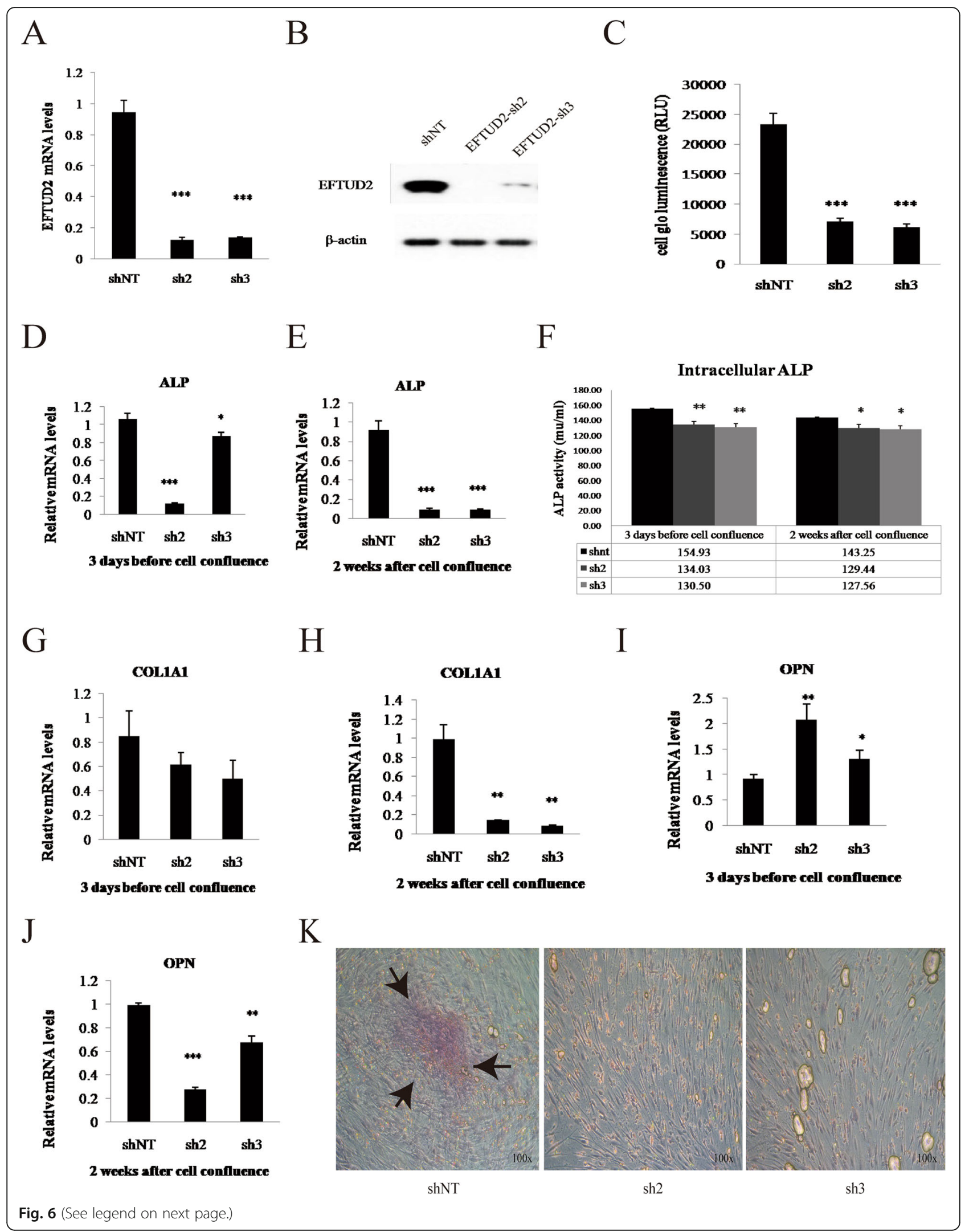


(See figure on previous page.)

Fig. 6 EFTUD2 gene knockdown in HCO cells. a Expression of EFTUD2 mRNA in HCO cells transfected with shNT, sh2, and sh3 lentiviruses. b Protein expression of EFTUD2 in HCO cells transfected with shNT, sh2, and sh3 lentiviruses. c Comparison of cell proliferation between HCO cells with EFTUD2 knockdown (sh2 and sh3) and the control group (shNT). d, e ALP mRNA levels in different groups (shNT, sh2, sh3) at 3 days before and 2 weeks after cell confluence in HCO cells. $\mathbf{f}$ Intracellular ALP activity (mu/ml) in HCO cells with EFTUD2 knockdown (sh2 and sh3) was much lower than in the control (shNT) at 3 days before cell confluence according to Student's $t$ test. $\mathbf{g}, \mathbf{h}$ COL1A1 mRNA levels in HCO cells in different groups (shNT, sh2, sh3) at 3 days before and 2 weeks after cell confluence. $\mathbf{i}, \mathbf{j}$ OPN mRNA levels in different groups (shNT, sh2, sh3) of HCO cells during development. $\mathbf{k}$ Alizarin red staining of $\mathrm{HCO}$ cells in these 3 groups at 3 days after cell confluence. Black arrows indicate calcific nodules ${ }^{*} P<0.05 ;{ }^{*} P<0.01 ;$ and ${ }^{* * *} P<0.001 ;$ Student's $t$ test)

the control group $(P<0.01)$ (Fig. 6g, h). The expression levels of $O P N$, a marker of mineralization during the late differentiation of the cellular matrix, in HCO cells transfected with sh2 and sh3 were 2.3- and 1.4-fold higher $(P<0.01$ and $P<0.05)$ than that in shNT-treated cells at 3 days before cell confluence, suggesting that $\mathrm{HCO}$ cells mature early in the differentiation stage (Fig. 6i). Thereafter, OPN expression declined by $30 \%$ and $70 \%$ $(P<0.001, P<0.01)$ in the sh2 and sh3 groups, respectively, indicating the inhibition of differentiation in the late differentiation stage (Fig. 6j). Calcium deposition significantly and consistently decreased without evident calcific nodules in those EFTUD2 knockdown groups (Fig. 6k).

We also investigated the role of EFTUD2 in $\mathrm{HC}$-a cell proliferation via EFTUD2-targeting shRNAs (sh2 and sh3, Additional file 5: Figure S4A-C). Similar to HCO cells, HC-a cell proliferation was significantly downregulated by approximately 70\% $(P<0.001)$ (Additional file 5 : Figure S4C and $\mathrm{S} 4 \mathrm{H}) 3$ days before cell confluence. The expression levels of differentiation-related genes in $\mathrm{HC}$-a cells, including the collagen type 10 alpha 1 (COL10A1) and SRY-box 9 (SOX9) genes, were assayed; COL10A1 gene expression was observed to be significantly increased by approximately 2 -fold before cell confluence, after which it decreased at the differentiation stage (Additional file 5: Figure S4D, E). SOX9, the key transcription factor throughout cartilage formation, was significantly reduced throughout the proliferation and differentiation stages (Additional file 5: Figure S4F, G).

\section{EFTUD2 knockdown and knockout resulted in TP53 pathway activation in vitro and in vivo}

A total of 997 differentially expressed genes were identified by RNA sequencing in HCO cells (Fig. 7a and Additional file 6: Figure S5A, B), and 20 genes showing the closest relationships were selected through $\mathrm{GO}$ and KEGG enrichment analysis (Fig. 7b, c). The cell cycle arrest and apoptosis genes were the most strongly affected in $\mathrm{HCO}$ cells after EFTUD2 gene disruption (Fig. 7b), while the TP53 pathway was suggested to be the most significant pathway according to KEGG analysis (Fig. 7c). Among the 6 genes involved in the TP53 (Tumor protein p53) downstream pathway, 5 genes, including $F A S$
(Fas cell surface death receptor), STEAP3 (STEAP3 metalloreductase), CASP3 (Caspase-3), P21 (Cyclindependent kinase inhibitor), and SESN1 (STEAP3 metalloreductase), were upregulated, whereas the THBS (Sestrin 1) gene was downregulated (Additional file 6: Figure S5C). STRING Protein-Protein Interaction Network analysis suggested that the EFTUD2 gene could affect cell growth through CASP3 (Fig. 7d).

We then analyzed the profiles of FAS, STEAP3, CASP3, P21, and SESN1 after EFTUD2 gene intervention and observed a significant increase of approximately 1.9to 6.7-fold over baseline for FAS, CASP3, STEAP3, P21, and SESN1 at 3 days before cell confluence in $\mathrm{HCO}$ cells (Fig. 8a-e). HCO cells with EFTUD2 knockdown (sh2) had higher protein levels of phosphorylated P53 compared with the shNT-transfected and nontransfected (control) groups $(P<0.05$, Fig. $8 f)$, whereas there was no difference between the negative control groups (shNT and control). Compared with that in the WT sample at $3 \mathrm{dpf}$, the expression of the P21, FAS, STEAP3, SESN1, and CASP3A genes in the $p 53$ pathway was 2- to 14.6fold higher $(P<0.01, P<0.01, P<0.05, P<0.001$, and $P<0.001$, respectively) in the homozygous eftud2 mutant zebrafish (Fig. 8g). CASP3B expression decreased to $2 \%$ of the WT expression level $(P<0.001)$. The protein expression of phosphorylated P53 (P-P53) was slightly elevated in the mutants with curved bodies at $4 \mathrm{dpf}(n=20$, $P=0.51$, Fig. 8 h), which may result from protein degradation. For further validation, we injected the $p 53$ morpholino into F3 generation (EF3) hybrids from eftud2 heterozygous mutated zebrafish (F2) and found that P53 knockdown could decrease the mortality of those curved larvae at $4 \mathrm{dpf}$ and $5 \mathrm{dpf}(P<0.05)$, similar to those with normal human EFTUD2 mRNA injected to a certain extent $(P<0.01$ and $P<0.001$, Fig. 8 i).

\section{Discussion}

By WES, a novel de novo frameshift EFTUD2 gene variant (c.1030_1031delTG, p.Trp344fs*2) was found in the first Chinese mandibulofacial dysostosis, Guion-Almeida type (MFDGA; MIM \#610536) patient with distinctive facial features, including arched eyebrows, broad nasal bridge, everted lips and micrognathia, low-set ears and external auditory canal stenosis, mild intellectual 


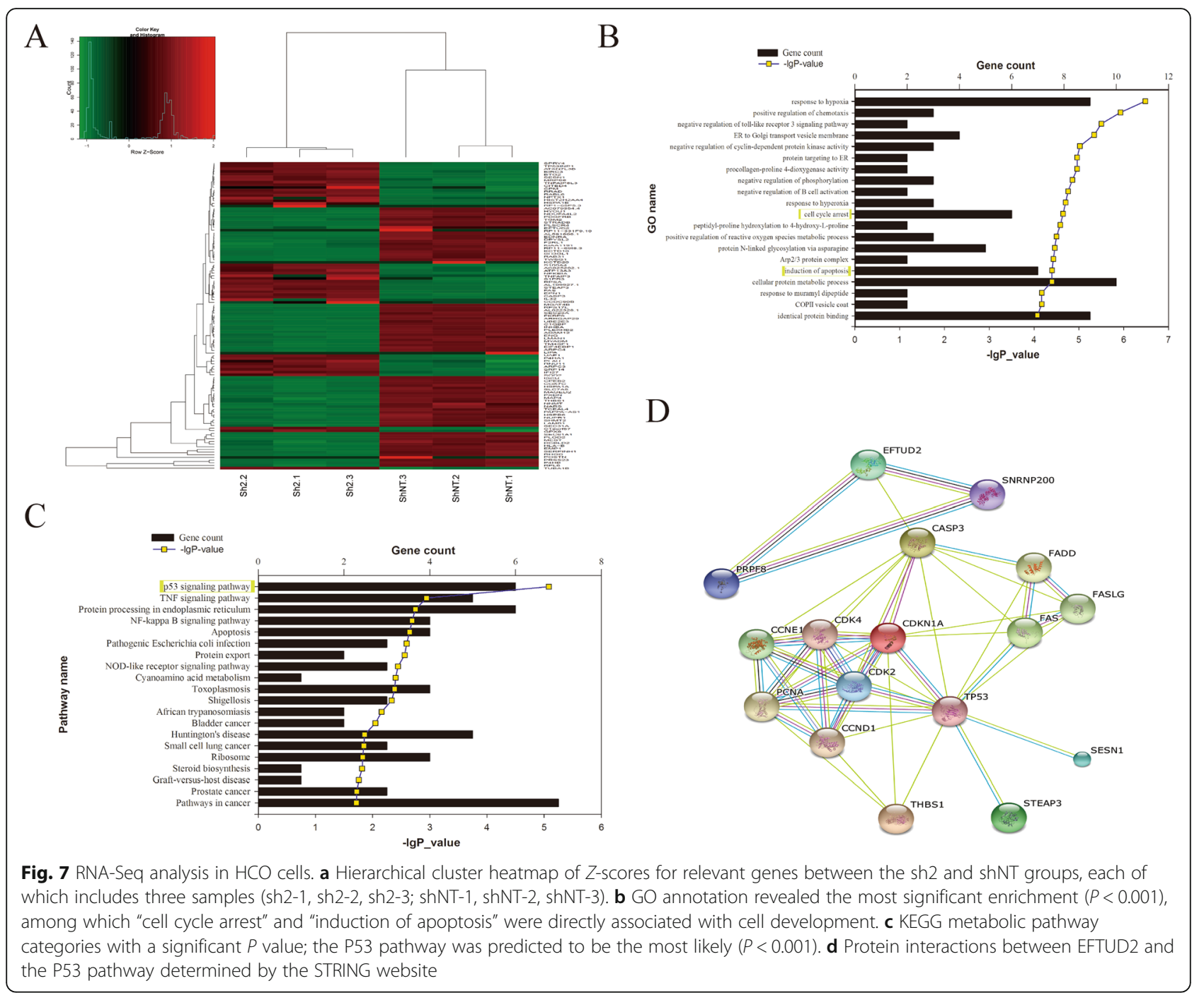

disability, and developmental delay. A 214.11-kb duplication in the chromosomal Xq28 region including the GABRA3, MIR105-1, MIR767, and MIR105-2 genes, identified in the proband via microarray analysis, was inherited from his healthy mother. Evidence from DGV indicated duplications of $197 \mathrm{~kb}$ (nssv655052), $222 \mathrm{~kb}$ (nssv657732), and $211 \mathrm{~kb}$ (nssv683872); these duplications were interpreted as likely benign, though a case (Patient 250326) reported in DECEPHER with a 371-kb duplication within the region had conditions of delayed speech, language development, and intellectual disability but without facial dysmorphological features [18]. Thus, the de novo deletion of TG resulting in the frameshift variant of EFTUD2 identified in the patient by WES would be pathogenic.

The widespread expression of eftud2 in mice at 11.5 dpf was previously observed to correlate with the affected region in patients [12], but there has been no description of eftud2 expression in the zebrafish model.
We observed a wide eftud2 expression pattern in zebrafish embryos at $24 \mathrm{hpf}$, and expression continued increasing until $48 \mathrm{hpf}$. In addition to small heads and curved bodies in the early development stage in eftud 2 -/- zebrafish larvae, which was consistent with a previous report [13], our knockout zebrafish model demonstrated other extra characteristics, including the absence of otoliths as well as bone and cartilage abnormalities. Synchrotron radiation X-ray microtomography showed that adult heterozygous F2-generation zebrafish exhibited a shortened jawbone and deformity of Meckel's cartilage, which mimic the phenotypes of human MFDM, suggesting the pathogenesis of EFTUD2 correlated with the craniofacial skeleton.

Craniofacial bone is formed by endochondral and intramembranous ossification. We generated EFTUD2 knockdown in two cell lines, $\mathrm{HCO}$ cells and $\mathrm{HC}$-a cells, to identify its influence on osteoblast proliferation. We observed a significant reduction in cell proliferation in 
A

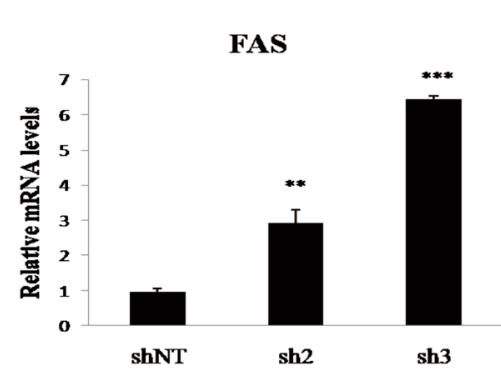

C

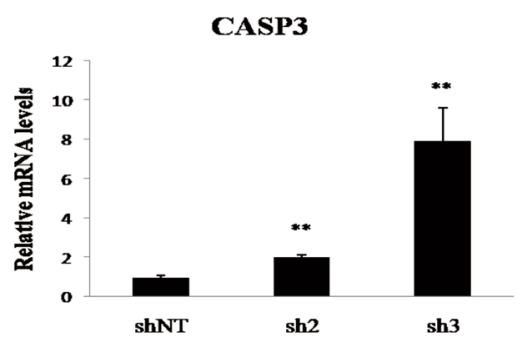

E

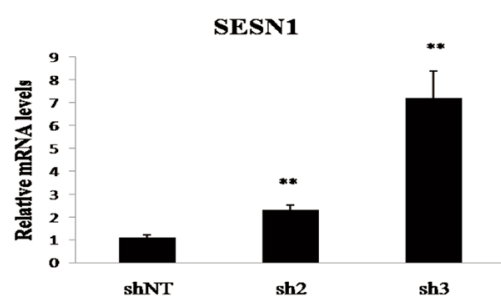

G

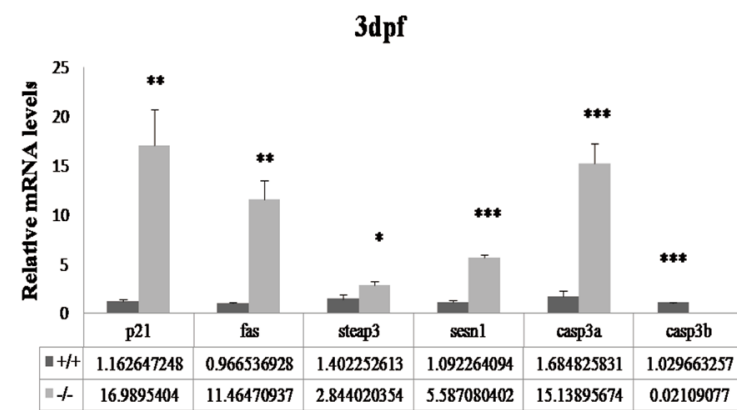

$\mathrm{H}$

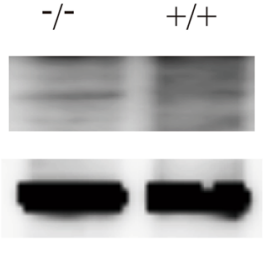

zebrafish (4dpf)
B

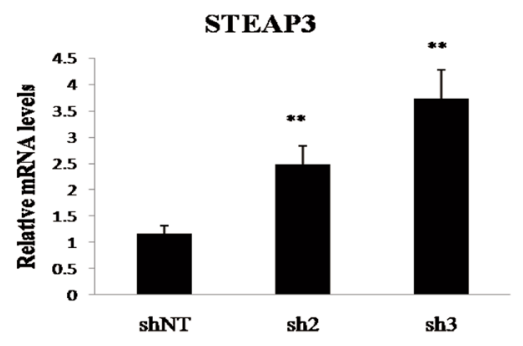

D

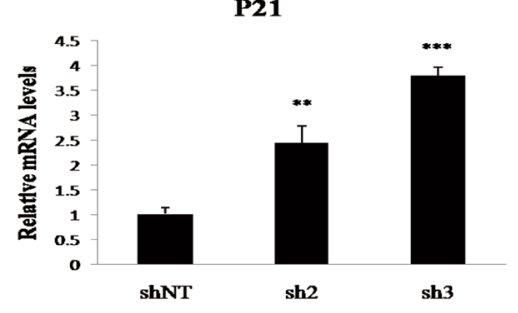

F

control shNT sh2
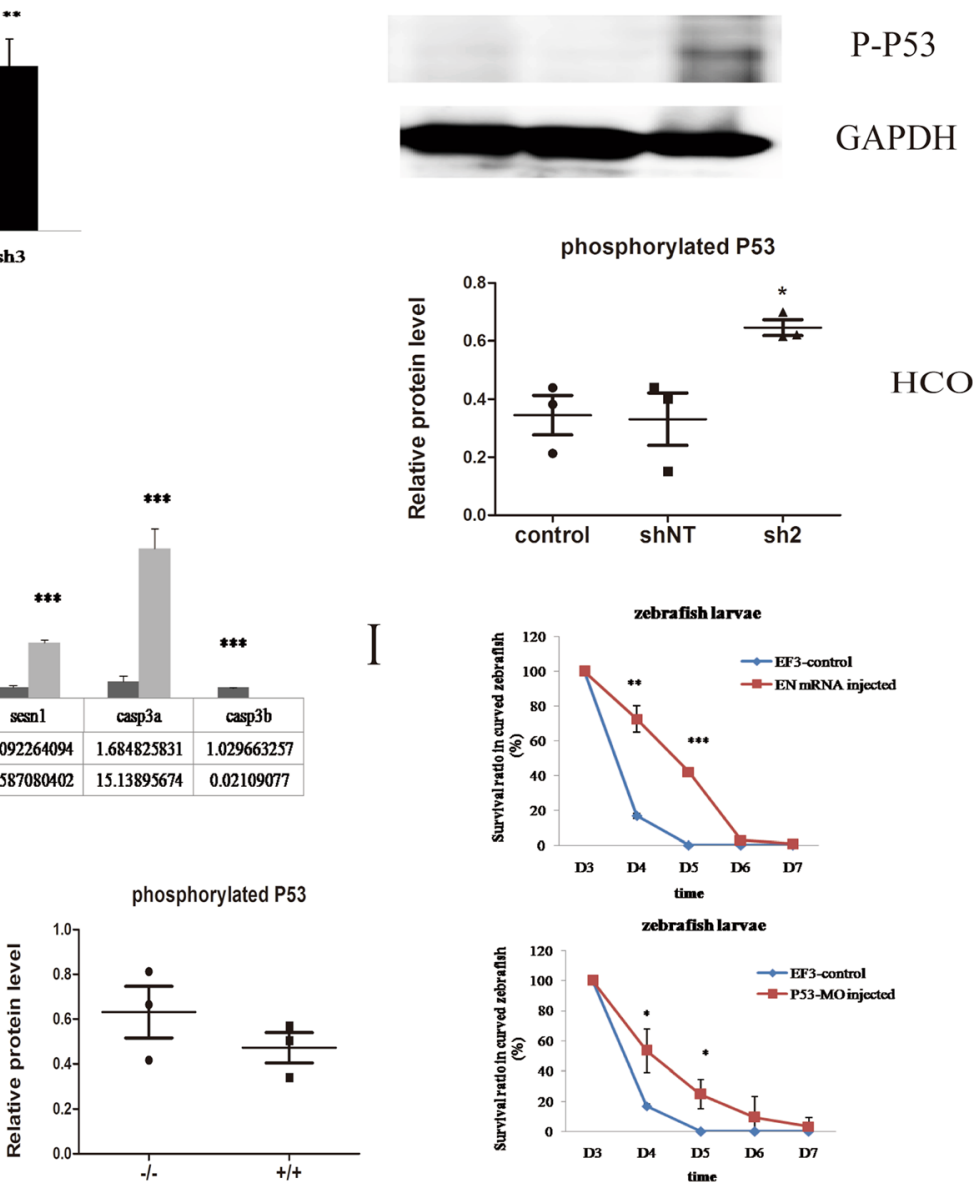

Fig. 8 (See legend on next page.) 
(See figure on previous page.)

Fig. 8 The p53 pathway mediates cell development in HCO cells and zebrafish. a Expression of the FAS gene in cells transfected with different lentiviruses (sh2, sh3, and shNT as a scramble control) at 3 days before cell confluence. HCO cells subjected to EFTUD2 knockdown (sh2 and sh3) exhibited increased FAS levels. $\mathbf{b}$ The expression of CASP3 in the sh2 and sh3 groups was dramatically increased compared with that in the shNT group. c STEAP3 mRNA levels in the sh2 and sh3 groups were markedly higher than that in the negative control. d P21 mRNA levels were also highly increased in the sh2 and sh3 groups. e SESN1 was highly expressed in the sh2 and sh3 groups. $\mathbf{f} H C O$ cells transfected with sh2 had a higher expression of phosphorylated P53 protein $(53 \mathrm{kDa})$ than the nontransfected (control) and shNT groups. $\mathbf{g}$ Expression of relevant genes involved in the P53 pathway in the EFTUD2 knockout (-/-) and WT (+/+) zebrafish. $\mathbf{h}$ The expression of phosphorylated P53 in EFTUD2-mutated larvae (-l-) with curved bodies and WT larvae at $4 \mathrm{dpf}$. $\mathbf{i}$ The survival rate among the curved F3 generation hybridizing from EFTUD2 heterozygous mutants (EF3 control), EF3 controls injected with EFTUD2 normal mRNA (EN mRNA), and p53 morpholino (P53-MO) during the early developmental stage $\left({ }^{*} P<0.05,{ }^{* *} P<0.01\right.$, and $\left.{ }^{* *} P<0.001\right)$

the two cell lines. The expression of $A L P$ and COL1A1, early differentiation markers $[19,20]$, was significantly decreased during the differentiation period in $\mathrm{HCO}$ cells. Furthermore, $O P N$, a marker of mineralization during the late differentiation of the cellular matrix, also reduced its expression by a maximum of $70 \%$. In addition, decreased calcium deposition and the formation of calcified nodules were found in HCO cells following EFTUD2 shRNA treatment, which highly suggests that HCO cell differentiation was prematurely stopped. Sox9, a key transcription factor throughout the formation of cartilage [21-24], also presented a significant reduction throughout the proliferation and differentiation stages in HC-a cells. These findings demonstrated that EFTUD2 knockdown could dramatically decrease the proliferation and inhibit the differentiation of $\mathrm{HCO}$ and $\mathrm{HC}$-a cells. EFTUD2 may play a role in maintaining normal calvarial osteoblast and chondrocyte proliferation.

High-throughput RNA sequencing revealed the TP53 signaling pathway was most strongly affected, and cell cycle arrest and apoptosis induction were significantly affected in EFTUD2 knockdown HCO cells. TP53, which is an important tumor suppressor gene, is responsible for cell growth, including the cell cycle, apoptosis, and angiogenesis $[25,26]$. A further experiment found increased phosphorylation of the TP53 protein, suggesting activation of the TP53 signaling pathway, which was validated in the knockout zebrafish model (4 dpf). Additionally, p53 knockdown definitely increased the survival of those mutated zebrafish larvae.

Furthermore, we identified increased expression in 5 out of the 6 major TP53-downstream cluster genes (FAS, STEAP3, CASP3, P21, and SESN1) in HCO cells, which was also validated in homozygotic eftud2 knockout zebrafish. Previous studies have shown that the TP53 signaling pathway is involved in inherited diseases with maxillofacial signs, such as Treacher Collins syndrome (TCS) or Acrofacial Dysostosis-Cincinnati type [27-29], neural stem cell apoptosis during embryonic development [30], and TP53-dependent apoptosis in neural progenitors in fn10a-mutated zebrafish [31]. Deml et al. reported a striking rise in apoptosis in many tissues in an eftud2-deficient zebrafish model [13].
Therefore, evidence of increasing TP53 signaling pathway activation was correlated with subsequent cell apoptosis, which could impact development, particularly ossification.

\section{Conclusions}

We identify a novel de novo frameshift EFTUD2 gene variant (c.1030_1031delTG, p.Trp344fs*2) in a Chinese MFDM patient. We established an EFTUD2 deficiency model in vitro and in vivo. Evidence of the transcriptome from cell lines and a zebrafish model suggested the TP53 signaling pathway was activated due to EFTUD2 disruption. Our findings also showed that the EFTUD2 gene could impact the proliferation and differentiation of osteoblasts and chondrocytes, suggesting that premature osteoblast and chondrocyte differentiation could be responsible for the pathogenesis of MFDM. Further studies on the specific mechanisms involved are necessary in the future.

\section{Supplementary information}

Supplementary information accompanies this paper at https://doi.org/10. 1186/s40246-019-0238-y.

Additional file 1. Materials and Methods including whole-genome aCGH analysis, whole-exome sequencing, embryo injection, luciferase single-strand annealing recombination analysis, DNA extraction and PCR for sequencing, real-time PCR and Western blotting. Table S1. Candidate genes with de novo variants in the proband. Table S2. Real-time PCR primers of relative genes in cells.

Additional file 2: Figure S1. eftud2 expression pattern in WT zebrafish. A: eftud2 expression in zebrafish embryos at $24 \mathrm{hpf}(\mathrm{a}, \mathrm{b}), 48 \mathrm{hpf}(\mathrm{c}, \mathrm{d})$, $3 \mathrm{dpf}(\mathrm{e}, \mathrm{f})$ and $5 \mathrm{dpf}(\mathrm{g}, \mathrm{h})$ was examined using whole-mount in situ hybridization over a period of 5 days, employing a specific eftud2 antisense probe; $(a, c, e, g)$ lateral view, $(b, d, f, h)$ dorsal view. B: Relative mRNA levels of eftud2 during the early developmental stages. C: Relative mRNA levels of eftud2 in adult zebrafish tissues.

Additional file 3: Figure S2. Targeting site and efficacy of eftud2targeted TALEN mRNAs. We also prepared three TALENs to construct the knockout zebrafish model, and the second one (T2) was the most effective for in vitro screening. A, B: The TALEN (T2) was designed at the first exon, which is presented in capital letters. C: In vitro efficacy was evaluated based on relative luciferase activity in TALEN-transfected Hek293T cells and the negative control. D: PCR products of eftud2 containing the target sequence could be digested by the T7E1 enzyme, in which the product from the mutant zebrafish was cleaved into two fragments, whereas that from WT zebrafish was intact. E: Sequencing results 
of F0 generation showed mixed signals from the target site, which may predict the combination with TALEN mRNA and the eftud2 gene.

Additional file 4: Figure S3. Zebrafish with eftud2 gene knockdown showed aberrant cartilage development. A, B: Larvae treated with an eftud2 morpholino (EMO) at 3dpf exhibited disrupted formation of Meckel's cartilage (a) and the ceratohyals (b) upon alcian blue and alizarin red staining compared with the WT fish, fish injected with a mismatch morpholino (EMIS-MO) and fish rescued with normal human EFTUD2 mRNA (Rescue). A shows the lateral view, and B shows the ventral view. C, D: Bone and cartilage staining among different groups of larvae (WT, EMO, rescue, EMIS-MO) also suggested abnormal cartilage development at 5 dpf.c, the ethmoid bones.

Additional file 5: Figure S4. EFTUD2 gene knockdown in HC-a. A: EXpression of EFTUD2 mRNA in $\mathrm{HC}$-a cells transfected with sh2 and sh3 lentivirus was lower than that in the shNT control.B: Protein expression of EFTUD2 decreased in $\mathrm{HC}$-a cells transfected with sh2 and sh3 lentivirus. C: Cell proliferation of $\mathrm{HC}$-a cells transfected with sh2 and sh3 lentivirus was disrupted compared with that of the shNT control. D-E: COL10A1 mRNA levels among different groups (shNT, sh2, sh3) of HC-a cells at 3 days before cell confluence, 3 days after cell confluence and 2 weeks after cell confluence. F-G: SOX9 mRNA levels in HC-a cells among different groups (shNT, sh2, sh3) at 3 days before and after cell confluence. H: Alcian blue staining of $\mathrm{HC}$-a cells among different groups (transfected with shNT, sh2 and sh3 lentiviruses). ${ }^{*}: P<0.05,{ }^{* *}: P<0.01,{ }^{* * *}: P<0.001$.

Additional file 6: Figure S5. Differentially expressed genes identified through RNA-Seq analysis. A: Correlation analysis between $\mathrm{HCO}$ cells transfected with shNT (Group1) or sh2 lentivirus (Group2), which showed a close relationship between the two groups. B: Genes of Group 1 and Group2 are located on all of the chromosomes. C: There were 6 genes involved in the P53 pathway, including P21, FAS, STEAP3, CASP3, SESN1 and THBS. All of these genes showed elevated expression, except THBS, which was downregulated.

\section{Abbreviations}

a-CGH: Array-CGH; ATP: Adenosine triphosphate; CASP3: Caspase-3; COL10A1: Collagen type 10 alpha 1; COL1A1: Collagen type 1 alpha 1; CT: Computerized tomography scan; DEGs: Differentially expressed genes; EF3: Hybridizing from heterozygous mutated zebrafish of F2-generation; EFTUD2: Elongation factor Tu GTP-binding domain-containing 2; EMISMO: eftud2 mismatch control morpholino; EMO: eftud2 morpholino; F1: Outcrossed F0 mosaic with wild-type zebrafish; F2: Obtained from eftud2 heterozygous mutants; FAS: Fas cell surface death receptor; FBP: Filtered back-projection; GO: Gene ontology; HC-a: Human articular chondrocyte; HCO: Human calvarial osteoblast; KEGG: Kyoto Encyclopedia of Genes and Genomes pathway; MFDM: Mandibulofacial dysostosis with microcephaly; MUP: 4-Methylumbelliferyl phosphate disodium salt; OPN: Osteopontin; P21: Cyclin-dependent kinase inhibitor; PBS: Phosphate buffer saline; PFA: Paraformaldehyde; P-P53: Phosphorylated P53; SESN1: STEAP3 metalloreductase; shNT: Scrambled shRNA; SNVs: Single nucleotide variants; SOX9: SRY-box 9; SR- $\mu$ CT: Synchrotron radiation X-ray microtomography; SSRF: Shanghai Synchrotron Radiation Facility; STEAP3: STEAP3 metalloreductase; STRING: Retrieval of Interacting Genes; TALEN: Transcription activator-like effector nucleases; THBS: Sestrin 1; TP53: Tumor protein p53; WES: Whole-exome sequencing; WISC: Wechsler Intelligence Scale for Children; WISH: Whole mount in situ hybridization; WT: Wild type

\section{Acknowledgements}

The authors thank all of the staff at the zebrafish laboratory of Children's Hospital at Fudan University and the School of Basic Medical Sciences, Fudan University, for their invaluable contributions to this project.

\section{Authors' contributions}

FL, YA and QL conceived and designed the study. YY participated in the study design and data analysis. YA performed WES sequencing and analyzed the data. JW carried out the in vitro and in vivo experiments, analyzed the data and wrote the manuscript. YH provided the scanning experiment. FL helped to interpret the results. XW participated in the experimental procedures. CS and WL contributed to the revision of the manuscript. FL and
YA revised and finalized the manuscript. All authors read and approved the final version of the manuscript.

\section{Funding}

This work is supported by National Natural Science Foundation of China (81873667).

\section{Availability of data and materials}

The datasets used and/or analyzed during the current study are available from the corresponding author upon reasonable request.

\section{Ethics approval and consent to participate}

This study was with by the approval of the faculty Ethics Committee of Children's Hospital of Fudan University, and written informed consent was obtained from the patient or his parents.

\section{Consent for publication}

The patient's consent was collected from his legal representatives for the publication of this study.

\section{Competing interests}

The authors declare that they have no competing interests.

\section{Author details}

'Department of Pediatric Endocrinology and Inherited Metabolic Diseases, Children's Hospital of Fudan University, Shanghai 201102, China. Institute of Pediatrics, Children's Hospital of Fudan University, Shanghai 201102, China. ${ }^{3}$ Shanghai Synchrotron Radiation Facility, Shanghai Institute of Applied Physics, Chinese Academy of Sciences, 239 Zhangheng Road, Pudong District, Shanghai 201204, China. ${ }^{4}$ Translational Medical Center for Development and Disease, Shanghai Key Laboratory of Birth Defect, Institute of Pediatrics, Children's Hospital of Fudan University, Shanghai 201102, China. ${ }^{5}$ Key Laboratory of Metabolism and Molecular Medicine, Ministry of Education, and Department of Biochemistry and Molecular Biology, School of Basic Medical Sciences, Fudan University, Shanghai 200032, China. ${ }^{6}$ Institute of Fudan-Minhang Academic Health System, Minhang Hospital, Fudan University, 170 Xinsong Road, Shanghai 201199, China. ${ }^{7}$ Human Phenome Institute, Fudan University, 825 Zhangheng Road, Shanghai 201203, China.

Received: 8 June 2019 Accepted: 13 September 2019

Published online: 05 December 2019

\section{References}

1. Matsuo M, Yamauchi A, Ito Y, et al. Mandibulofacial dysostosis with microcephaly: a case presenting with seizures. Brain and Development. 2017;39:177-81.

2. Huang L, Vanstone MR, Hartley T, et al. Mandibulofacial dysostosis with microcephaly: mutation and database update. Hum Mutat. 2016;37:148-54.

3. Guion-Almeida ML, Zechi-Ceide RM, Vendramini S, Tabith JA. A new syndrome with growth and mental retardation, mandibulofacial dysostosis, microcephaly, and cleft palate. Clin Dysmorphol. 2006;15:171-4.

4. Zarate YA, Bell C, Schaefer GB. Radioulnar synostosis and brain abnormalities in a patient with 17q21.31 microdeletion involving EFTUD2. Cleft Palate Craniofac J. 2015;52:237-9.

5. Voigt C, Megarbane A, Neveling K, et al. Oto-facial syndrome and esophageal atresia, intellectual disability and zygomatic anomalies expanding the phenotypes associated with EFTUD2 mutations. Orphanet J Rare Dis. 2013;8:110.

6. Wieczorek D, Shaw-Smith C, Kohlhase J, et al. Esophageal atresia, hypoplasia of zygomatic complex, microcephaly, cup-shaped ears, congenital heart defect, and mental retardation--new MCA/MR syndrome in two affected sibs and a mildly affected mother? Am J Med Genet A. 2007;143A:1135-42.

7. Smigiel $R$, Bezniakow $N$, Jakubiak $A$, et al. Phenotype analysis of Polish patients with mandibulofacial dysostosis type Guion-Almeida associated with esophageal atresia and choanal atresia caused by EFTUD2 gene mutations. J Appl Genet. 2015;56:199-204.

8. Luquetti DV, Hing AV, Rieder MJ, et al. "Mandibulofacial dysostosis with microcephaly" caused by EFTUD2 mutations: expanding the phenotype. Am J Med Genet A. 2013;161A:108-13. 
9. Lehalle $D$, Gordon $C T$, Oufadem $M$, et al. Delineation of EFTUD2 haploinsufficiency-related phenotypes through a series of 36 patients. Hum Mutat. 2014;35:478-85.

10. Lines MA, Huang L, Schwartzentruber J, et al. Haploinsufficiency of a spliceosomal GTPase encoded by EFTUD2 causes mandibulofacial dysostosis with microcephaly. Am J Hum Genet. 2012;90:369-77.

11. Yu K, Luk HM, Gordon CT, et al. Mandibulofacial dysostosis Guion-Almeida type caused by novel EFTUD2 splice site variants in two Asian children. Clin Dysmorphol. 2018;27:31-5.

12. Gordon CT, Petit F, Oufadem M, et al. EFTUD2 haploinsufficiency leads to syndromic oesophageal atresia. J Med Genet. 2012;49:737-46.

13. Deml B, Reis $L M$, Muheisen S, Bick D, Semina EV. EFTUD2 deficiency in vertebrates: identification of a novel human mutation and generation of a zebrafish model. Birth Defects Res A Clin Mol Teratol. 2015;103:630-40

14. Robu ME, Larson JD, Nasevicius A, et al. p53 activation by knockdown technologies. PLoS Genet. 2007;3:e78.

15. Vecchione L, Gambino V, Raaijmakers J, et al. A vulnerability of a subset of colon cancers with potential clinical utility. Cell. 2016;165:317-30.

16. Wu J, Yang Y, Sun C, et al. Disruption of the gaa gene in zebrafish fails to generate the phenotype of classical Pompe disease. DNA Cell Biol. 2017;36:10-7.

17. Walker MB, Kimmel CB. A two-color acid-free cartilage and bone stain for zebrafish larvae. Biotech Histochem. 2007:82:23-8.

18. Shaikh TH, Gai X, Perin JC, et al. High-resolution mapping and analysis of copy number variations in the human genome: a data resource for clinical and research applications. Genome Res. 2009;19:1682-90.

19. Fantner GE, Hassenkam T, Kindt JH, et al. Sacrificial bonds and hidden length dissipate energy as mineralized fibrils separate during bone fracture. Nat Mater. 2005;4:612-6.

20. Aubin JE, Liu F, Malaval L, Gupta AK. Osteoblast and chondroblast differentiation. Bone. 1995;17:77S-83S.

21. Dy P, Wang W, Bhattaram P, et al. Sox9 directs hypertrophic maturation and blocks osteoblast differentiation of growth plate chondrocytes. Dev Cell. 2012:22:597-609.

22. Wuelling M, Vortkamp A. Chondrocyte proliferation and differentiation. Endocr Dev. 2011:21:1-11.

23. Akiyama H, Chaboissier MC, Martin JF, Schedl A, de Crombrugghe B. The transcription factor Sox9 has essential roles in successive steps of the chondrocyte differentiation pathway and is required for expression of Sox 5 and Sox6. Genes Dev. 2002;16:2813-28.

24. Kolettas E, Muir HI, Barrett JC, Hardingham TE. Chondrocyte phenotype and cell survival are regulated by culture conditions and by specific cytokines through the expression of Sox-9 transcription factor. Rheumatology (Oxford). 2001;40:1146-56.

25. Kim MP, Zhang Y, Lozano G. Mutant p53: multiple mechanisms define biologic activity in cancer. Front Oncol. 2015;5:249.

26. Wang $X$, Simpson ER, Brown KA. p53: protection against tumor growth beyond effects on cell cycle and apoptosis. Cancer Res. 2015;75:5001-7.

27. Watt K, Neben CL, Hall S, Merrill AE, Trainor PA. tp53-dependent and independent signaling underlies the pathogenesis and possible prevention of Acrofacial Dysostosis - Cincinnati type. Hum Mol Genet. 2018;27:2628.

28. Trainor PA. Craniofacial birth defects: the role of neural crest cells in the etiology and pathogenesis of Treacher Collins syndrome and the potential for prevention. Am J Med Genet A. 2010;152A:2984-94.

29. Trainor PA, Dixon J, Dixon MJ. Treacher Collins syndrome: etiology, pathogenesis and prevention. Eur J Hum Genet. 2009;17:275-83.

30. Jones NC, Lynn ML, Gaudenz K, et al. Prevention of the neurocristopathy Treacher Collins syndrome through inhibition of p53 function. Nat Med. 2008;14:125-33.

31. Lei L, Yan SY, Yang R, et al. Spliceosomal protein eftud2 mutation leads to p53-dependent apoptosis in zebrafish neural progenitors. Nucleic Acids Res. 2017:45:3422-36.

\section{Publisher's Note}

Springer Nature remains neutral with regard to jurisdictional claims in published maps and institutional affiliations.

\section{Ready to submit your research? Choose BMC and benefit from:}

- fast, convenient online submission

- thorough peer review by experienced researchers in your field

- rapid publication on acceptance

- support for research data, including large and complex data types

- gold Open Access which fosters wider collaboration and increased citations

- maximum visibility for your research: over $100 \mathrm{M}$ website views per year

At BMC, research is always in progress.

Learn more biomedcentral.com/submissions 